Pesq. Vet. Bras. 29(4):317-321, abril 2009

\title{
Ultra-sonografia do fígado, aparelho renal e reprodutivo da jibóia (Boa constrictor) ${ }^{1}$
}

\author{
Francisco C.P. Neto ${ }^{2}$, Porfírio C. Guerra ${ }^{3}$, Francisco B. Costa ${ }^{4}$, Adriana V.C. Araújo², \\ Maria A. Miglino ${ }^{5}$, Pedro P. Bombonato ${ }^{5}$, Luiz C. Vulcano ${ }^{6}$ e Flávio R. Alves ${ }^{7 *}$
}

\begin{abstract}
Neto F.C.P., Guerra P.C., Costa F.B., Araújo A.V.C., Miglino M.A., Bombonato P.P.,Vulcano L.C. \& Alves F.R. 2009. [Ultrasonography of the liver, renal and reproductive apparatus of Boa constrictor Snake.] Ultra-sonografia do fígado, aparelho renal e reprodutivo da jibóia (Boa constrictor). Pesquisa Veterinária Brasileira 29(4):317-321. Departamento de Cirurgia, Faculdade de Medicina Veterinária e Zootecnia, Universidade de São Paulo, Av. Prof. Dr. Orlando Marques de Paiva 87, São Paulo, SP 05508-270, Brazil. E-mail: flaviovet@usp.br

Currently, wild animals have been received special attention for domestic breeding. In Wild Animal Medicine, ultrasonographic exams could be considered as a tool to diagnose and prevent disease. Ultrasonographic examination of twenty snakes (Boa constrictor) was performed in order to describe the morphological and ultrasonography appearance of their coelomic structures. Examination by ultrasonography revealed the parechymal liver ranging from slightly hypoechogenic to hyperechogenic, with echogenic margins and homogeneous echotexture in total scanning of this organ. The kidney was accessible for examination by sagittal ultrasound, showing an ellipsoid shape, a hyperechogenic capsule with a thin and regular wall. Ultrasonographic scanning of ovarian follicle showed an ovoid shape, as well as a thin, regular and slightly hyperechogenic edge. No evident structures from male reproductive system were identified by ultrasound examination because of their similar echogenic appearance to the tissues around and the "body fat" that lies in this region. Examination by ultrasound of the coelomic structures was a fast and repeatable technique, which provides useful data to recognize the morphology, sintopy and ultrasonographic appearance of organs such as liver, kidneys and vitellogenic follicles.
\end{abstract}

INDEX TERMS: Boa constrictor snake, ultrasound, wild animals, Amazon.

\footnotetext{
${ }^{1}$ Recebido em 14 de julho de 2008.

Aceito para publicação em 12 de janeiro de 2009.

${ }^{2}$ Curso de Medicina Veterinária (CMV), Universidade Estadual do Maranhão (UEMA), Cidade Universitária Paulo VI, Tirirical, Cx. Postal 9, São Luís, MA 65055-970, Brasil.

${ }^{3}$ Departamento de Clínicas, CMV, UEMA, São Luís, MA.

${ }^{4}$ Pós-Graduando em Ciências Veterinárias, CMV, UEMA, São Luiz, MA.

${ }^{5}$ Departamento de Cirurgia, Faculdade de Medicina Veterinária e Zootecnia (FMVZ), Universidade de São Paulo (USP), Av. Prof. Dr. Orlando Marques de Paiva 87, São Paulo, SP 05508-270, Brasil.

${ }^{6}$ Departamento de Radiologia e Reprodução Animal, Faculdade de Medicina Veterinária e Zootecnia (FMVZ), Universidade Estadual Paulista (Unesp), Rua Tiradentes 67, Botucatu, São Paulo, SP 18611-840, Brasil.

7 Disciplina Diagnóstico por Imagem, Curso de Medicina Veterinária, Universidade Federal do Piauí (UFPI), Campus Cinobelina Elvas, BR125 Km 3, Planalto Cibrazem, Bom Jesus, Piauí, PI 64900-000, Brasil. "Autor para correspondência: flaviovet@usp.br
}

RESUMO.- Atualmente os animais silvestres têm despertado o interesse particular na criação domestica. Na medicina de animais selvagens, os exames ultra-sonográficos podem ser considerados como ferramenta para diagnosticar e prevenir doenças. Deste modo, realizou-se um estudo em 20 jibóias (Boa constrictor), a fim de caracterizar a morfologia e aparência ultra-sonográfica das estruturas presentes da cavidade celomática desses animais. Ultra-sonograficamente, o fígado apresentou-se variando de hipoecóica a levemente hiperecogênica, com margens ecogênicas e ecotextura homogênea em toda sua extensão. Os rins mostraram formato elipsóide, com cápsula fina, regular e hiperecóica. Os folículos ovarianos apresentaram formato ovóide, margens finas, regulares e discretamente hiperecóicas. As estruturas do sistema reprodutor do macho não foram evidenciadas com precisão, devido a sua ecogenicidade similar em relação às 
estruturas adjacentes e pela presença do "corpo gorduroso" localizado nessa região. A ultra-sonografia da cavidade celomática em jibóias demonstrou ser uma técnica rápida e de fácil acesso, permitindo identificar a morfologia, sintopia e aparência ultra-sonográfica de estruturas como o fígado, rins e de folículos vitelogênicos nas fêmeas.

TERMOS DE INDEXAÇÃO: Jibóia, ultra-sonografia, animais silvestres, Amazônia.

\section{INTRODUÇÃO}

Ao longo dos anos, uma grande variedade de espécies selvagens tem sido domesticada. Nos Estados Unidos da América aproximadamente vinte milhões de norte-americanos são criadores de algum tipo de réptil, em sua maioria serpentes e dentre elas as jibóias (Das \& Banerjee 2004). A combinação de um comportamento tranqüilo em cativeiro, associado ao tamanho e seu padrão estético, tem influenciado no aumento da procura comercial destes animais. Esse fato tem aumentado a necessidade de um maior contingente de informações em relação a esta espécie (Loizou et al. 2007).

Estas serpentes habitam um vasto território nas Américas Central e do Sul, sendo mais encontradas nas florestas densas da Costa Rica e em toda a floresta Amazônica (Nogueira et al. 2003).

Segundo Fordham et al. (2007) e Todd \& Andrews (2008), as jibóias (Boa constrictor) apresentam um tamanho variável, podendo chegar a quatro metros de comprimento, com corpo cilíndrico e ligeiramente comprimido nas laterais, evidenciando uma forte musculatura constritora. O sistema de acasalamento conforme descritos por Isaza et al. (1993); Bertona \& Chiaraviglio (2003) e Chiaraviglio et al. (2003) é poligâmico.

Os avanços alcançados na medicina de animais selvagens têm possibilitado uma extensa gama de exames complementares visando padronizar valores de referência que auxiliem em avaliações confiáveis, como as das funções hepática e renal (De Sousa-e-Silva et al. 2003).

A difusão de técnicas minimamente invasivas de diagnóstico por imagem, para a avaliação de espécies selvagens já constitui uma realidade (Alves et al. 2007). Autores como Isaza et al. 1993a, 1993b têm descrito a cavidade celomática nesta espécie como importante colaboradora no equilíbrio homeostático da espécie, particularmente pela deposição de gordura observada pela presença do "corpo gorduroso".

Ultra-sonograficamente, tal estrutura tem sido identificada com maior ou menor frequência, segundo o habitat e território ocupado pelo animal. Quando presente muitas vezes dificulta a visualização de orgãos importantes presentes na cavidade celomática. Nesse contexto, a avaliação ultra-sonográfica da cavidade celomática e a utilização de biopsias guiadas por ultra-som na jibóia ( $B$. constrictor) se traduzem na possibilidade de incremento do conhecimento, tanto da morfologia, quanto da aparência ultrasonográfica normal nessa espécie silvestre, contribuindo diretamente para a manutenção de sua conservação.

\section{MATERIAL E MÉTODOS}

\section{Animais}

Foram utilizadas 20 jibóias, sendo 10 fêmeas e 10 machos. As fêmeas apresentaram comprimento médio de $\pm 2,35 \mathrm{~m}$ de comprimento, enquanto os machos mediram $\pm 1,80 \mathrm{~m}$. As idades não foram determinadas, visto que os animais foram provenientes de apreensões e capturas realizadas pelo CETAS (Centro de Triagem de Animais Silvestres) do Estado do Maranhão. A literatura tem relatado um comprimento médio para jibóias adultas em torno de 2,50m. Os animais foram mantidos em caixas plásticas simples, de tamanho adequado, e alimentadas com camundongos fornecidos pelo Biotério da Universidade Estadual do Maranhão (UEMA), São Luís, MA. Para a identificação do sexo durante o exame ultra-sonográfico, buscou-se a presença de "espículas" para-cloacais presentes nos machos da espécie, procedimento realizado sem grandes dificuldades.

\section{Procedimento anestésico}

Para a realização dos exames, os animais foram submetidos à contenção química utilizando-se cloridrato de quetamina a $5 \%$ na dosagem de $40 \mathrm{mg} / \mathrm{kg}$ associado a diazepan, na dosagem de $2 \mathrm{mg} / \mathrm{kg}$, por via intramuscular, aplicada na região dorsal, próximo aos processos espinhosos. Em seguida os animais foram devolvidos às caixas de contenção, iniciando-se o exame ultra-sonográfico 15 minutos depois de verificada sedação adequada, caracterizada pelo déficit do reflexo lingual e relaxamento muscular. A associação anestésica utilizada neste protocolo promoveu um tempo hábil de 45 minutos para realização dos exames, não havendo, necessidade de reaplicações das drogas.

\section{Exame ultra-sonográfico}

Foram realizados no Setor de Diagnóstico por imagem do Hospital Veterinário da Universidade Estadual do Maranhão, São Luís. Os animais foram dispostos em decúbito dorsal sob mesa própria, de uso padrão em exames ultra-sonográficos. O estudo ultra-sonográfico seguiu-se com a varredura da cavidade celomatica das jibóias, desde a porção mais proximal, demarcada pela imagem cardíaca e grandes vasos até a sua porção mais distal, demarcada pelo estreitamento abrupto do seu diâmetro corpóreo, a fim de visualizar e caracterizar algumas estruturas presentes nessa cavidade. Para isso, utilizou-se um aparelho de ultra-som portátil PIE MEDICAL Falco Vet. e um transdutor convexo multifrequencial, cuja freqüência variava entre 5 e $7,5 \mathrm{MHz}$. Como meio de contato para transmissão de ultra-sons foi utilizado o Carbogel $\AA_{\text {. }}$.

Como janela ultra-sonográfica hepática, utilizaram-se as bases de referência padronizadas por (Isaza, et al. 1993), no qual se promoveu uma tentativa de identificação do parênquima, na linha ventro-lateral direita, caudalmente ao ápice cardíaco e cranialmente ao estômago.

$\mathrm{Na}$ tentativa de identificação das silhuetas renais, o transdutor foi posicionado cranialmente à cloaca, na porção final da cavidade celomática, no qual promoveu-se a varredura na parede lateral da cavidade, por cortes sargitais.

Nos machos, procurou-se detectar os testículos, adjacentemente cranial aos rins. Sendo que nas fêmeas, em topografia semelhante os folículos foram pesquisados na tentativa de sua identificação. Foram acessados por cortes sagitais no terço caudal da cavidade celomática, cranial aos rins. Em topografia semelhante também foram acessados os folículos ovarianos, nas fêmeas, quando presentes. 


\section{RESULTADOS}

Em todos os animais, o fígado foi visualizado no terço médio da cavidade celomática, como uma estrutura cuja ecogenicidade variou de hipoecóica a levemente hiperecogênica, com margens ecogênicas, apresentando ecotextura homogênea em toda sua extensão e ocupando grande parte do terço médio direito da cavidade celomática, quando comparado à gordura adjacente (Fig.1a), seguindo-se pela janela acústica previamente preconizada. As veias cava caudal e porta hepática foram evidenciadas como estruturas calibrosas, distinguidas pela maior ecogenicidade da parede portal, ambas dispostas sob o mesmo plano de varredura (Fig.1b). A vesícula biliar não foi visualizada sob os planos de varredura realizados.

Avaliação ultra-sonográfica renal seguiu-se a partir da sua identificação no terço médio-caudal do segmento cor-
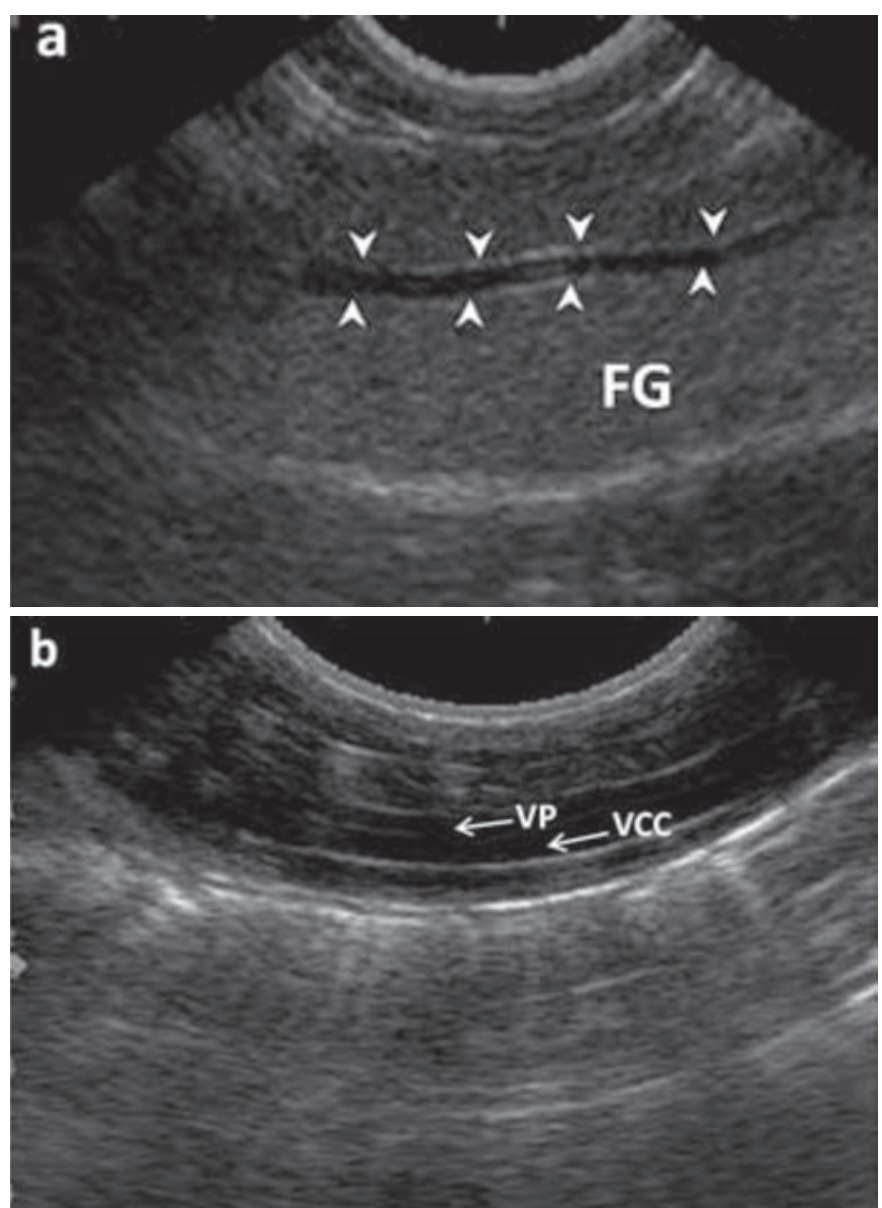

Fig.1. (a) Imagem ultra-sonográfica sagital do terço médio da cavidade celomática da jibóia (Boa constrictor), demonstrando variação de ecogenicidade do parênquima hepático [FG] (hipoecóico a levemente hiperecogênico). Suas margens são ecogênicas e sua ecotextura é homogênea em toda sua extensão, exceto pela presença da veia porta centralmente, distinguida pela maior ecogenicidade de sua parede (setas). (b) A veia porta (VP) pode ser visualizada sob o mesmo plano da veia cava caudal (VCC).
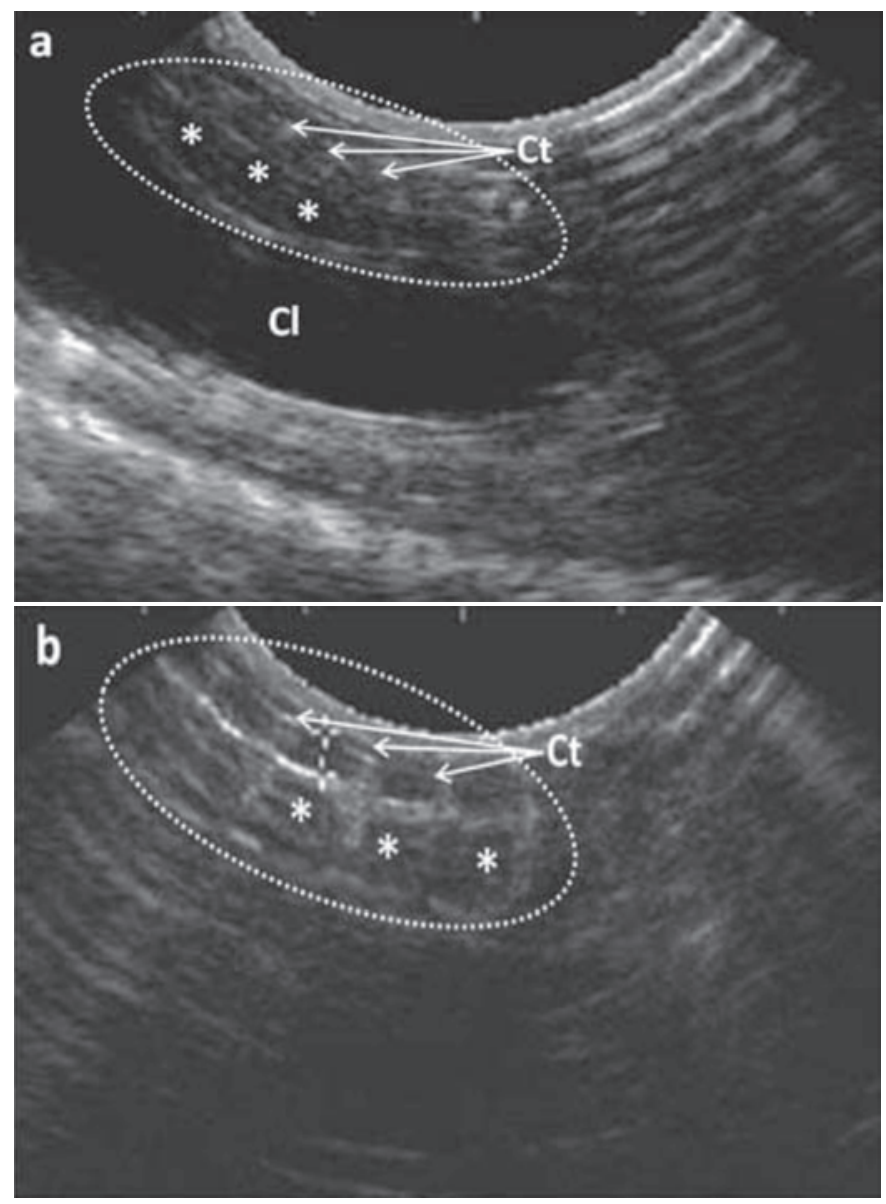

Fig.2. (a) e (b) Imagem ultra-sonográfica sagital do terço caudal da cavidade celomática da jibóia (Boa constrictor), demonstrando rins de formato elipsóide (círculo pontilhado), achatados dorso-ventralmente e segmentados. Sua cápsula apresenta parede fina, regular e hiperecóica. A cortical renal $\left(^{*}\right)$ é vista mais hiperecóica, comparada a medular hipoecóica (setas).

poral dos animais estudados e dorso-lateral ao cólon, esses foram também identificados em todos os animais. Os rins mostraram formato elipsóide, com o rim direito maior e posicionado mais cranialmente, quando comparado ao esquerdo. Caracterizaram-se como estruturas bem definida, achatados dorso-ventralmente, segmentados e ligeiramente envolvido pelo "corpo gorduroso", hiperecóico (Fig.2a,b). Ultra-sonograficamente, apresenta cápsula de parede fina, regular e hiperecóica. A junção cortico-medular mostrou-se distinguível. A córtex renal foi vista mais ecogênica, quando comparada à região medular, no qual se encontram os ductos coletores e canalículos renais, conseqüentemente mais hipoecogênica. Uma linha central hiperecogênica é vista correspondendo à área de deposição da gordura da pelve renal.

Em todas as fêmeas estudadas, os folículos ovarianos pesquisados na região de cavidade celomática foram passiveis de identificação próxima à topografia renal. Estes apresentaram formato ovóide, preenchidos basicamente por conteúdo anecóico, com ou sem septações hiperecóicas 


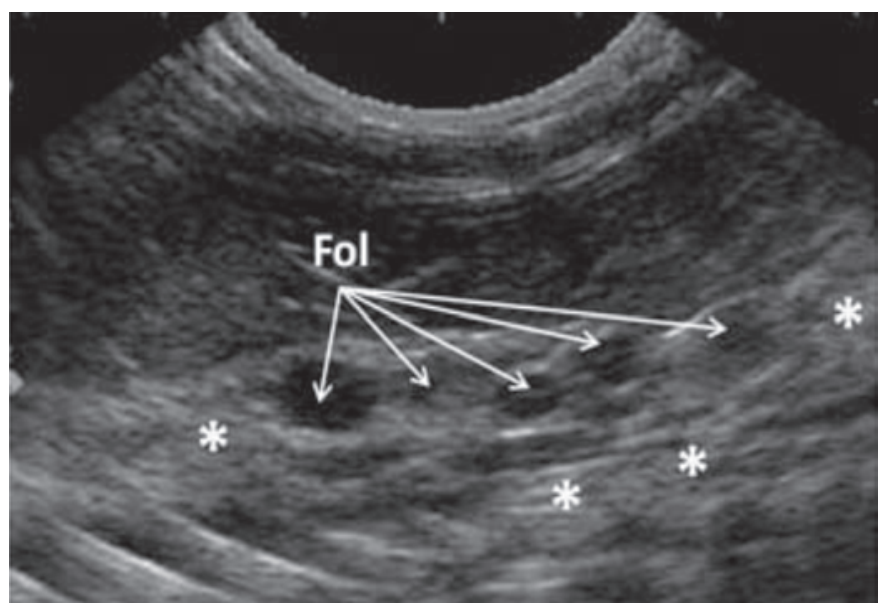

Fig.3. Imagem ultra-sonográfica sagital do terço caudal da cavidade celomática da jibóia (Boa constrictor), demonstrando folículos ovarianos (Fol) de formato ovóide, bordas finas, regulares, hiperecóicas e com ecotextura homogênea e grosseira. Chama a atenção seu aspecto semelhante a um "colar de contas". O "corpo gorduroso" $\left({ }^{*}\right)$ pode ser visto como uma extensa área que envolve os folículos.

centrais, com margens finas, regulares, e com ecotextura e grosseira, cujo padrão variou de hipoecogênico à levemente hiperecóico. Seu aspecto assemelhava-se a um "colar de contas" e mostraram-se envolvido por uma extensa porção ecogênica, provavelmente do "corpo gorduroso" (Fig.3). Nos machos os testículos foram identificados em apenas três animais estudados, não sendo possível, contudo, a delimitação de suas dimensões. Quando observados, os testículos foram acessados no terço caudal da cavidade celomática, cranialmente aos rins, hipoecogênicos, em meio à gordura peri-renal.

\section{DISCUSSÃO}

Os órgãos avaliados na jibóia pelo exame ultra-sonográfico transcutâneo, apresentaram níveis de ecotextura que permitiram resolução adequada para a varredura de órgãos como fígado, rins, folículos ovarianos. De modo semelhante, Chetboul et al. (2004) também evidenciaram a aparência ultra-sonográfica de estruturas componentes da cavidade celomática em cobras. O uso de gel de acoplamento acústico mostrou-se satisfatório, proporcionando acoplamento acústico adequado e minimizando a presença de artefatos de técnica durante a avaliação.

Os exames ultra-sonográficos avaliados em tartarugas por Penninck et al. (1991) e em cobras por Snyder et al. (1999) descreveram achados referentes a imagem de grandes vasos nesses animais. Corroborando com as descrições desses autores, neste estudo foi possível uma varredura e identificação topográfica precisa das veias cava caudal e porta hepática.

Neste estudo, o exame ultra-sonográfico evidenciou o fígado da jibóia, em todos os animais, com um formato fusiforme, percorrido centralmente por duas veias, que correspondem topograficamente às veias cava caudal e porta hepática. Corroborando com esta informação, estudos realizados por Bragdon (1953a,b) descrevendo a anatomia cirúrgica em cobras aquáticas, demonstraram o fígado como a estrutura mais evidente dentre as vísceras da cavidade celomática. A inabilidade para a observação da vesícula biliar, ultra-sonograficamente, deve-se provavelmente ao seu obscurecimento por gás excessivo em alças intestinais. De igual modo, também Isaza et al. (1993a) descreveram a influência do preenchimento do aparelho digestório como um fator negativo para visualização de órgãos da cavidade celomática, tais como pâncreas e baço.

Corroborando com este achado, Gartrell et al. (2002) e Martinez-Torres et al. (2006) em estudos não-invasivos em lagartos, também identificaram como característica ultrasonográfica principal do fígado a variação de hipo a levemente hiperecogênico, cuja ecotextura, e sua situação percutânea, fazem desse órgão uma janela acústica de eleição para a identificação e avaliação de estruturas adjacentes. Aliado a isso, o padrão ecogênico do tecido adiposo de sua cápsula favoreceram a determinação precisa de seus limites.

A localização dorso-lateral dos rins em relação ao cólon e no terço médio-caudal do segmento corporal observada em todos os espécimes examinados por Bragdon (1953) também foi verificada por Isaza et al. (1993a,b) e em nosso estudo. Semelhantemente, esses autores também descreveram os rins da jibóia mais hipoecóicos em relação à gordura peri-renal. Assim como previamente comentado, apesar de serem identificados em todos os animais desse estudo, foi constante a dificuldade de visualização detalhamento das estruturas renais como conseqüência do "corpo gorduroso", limitando a riqueza de detaIhes e limites desse órgão. Bragdon (1953), em serpentes, e Sainsbury \& Gili (1991), em lagartos, já descreviam a presença de dois corpos gordurosos localizados ventralmente à cavidade celomática, cujo tamanho depende do estado reprodutivo ou nutricional do animal, dificultando o acesso de órgãos situadas adjacentes a esta estrutura.

As informações desses autores foram confirmadas, quando os animais do experimento foram submetidos a um período de jejum que variou de 20 a 30 dias, a partir do qual houve a observação mais detalhada de órgãos localizados adjacentes ao corpo gorduroso, tais como o rim. De modo geral, devido ao fato das jibóias estudadas serem provenientes de capturas em regiões cuja fauna silvestre é rica em pequenos animais, acreditamos que a dieta anterior à captura possa influenciar nos resultados obtidos, visto que alguns animais mostraram corpo gorduroso mais exuberante do que outros, o que influenciaria na detecção de tais estruturas. Esses achados também são confirmados pelas descrições realizadas por Gitirana (1987), Ibarguengoytia \& Cussac (1996), Roig et al. (2000), O'Donnell et al. (2004), Santos \& Llorente (2004), Bizerra et al. (2005), Madsen et al. (2006) e Bradshaw et al. (2007), demonstrando o efeito nutricional sobre a formação do corpo gorduroso e visualização de estruturas internas em répteis. 
A visualização adequada dos folículosovarianos sofre influência do ciclo reprodutivo, conforme descrições de Bizerra et al. (2005), Martinez-Torres et al. (2006) e (Gilman \& Wolf (2007). Quando visualizados, os folículos apresentaram aparência ultra-sonográfica definidas como estruturas anecóicas com margens anecogênicas e conteúdo parenquimal misto, particularmente pelo acúmulo de vitelo, semelhante ao que foi observado por Rostal \& Robeck (1989) e por Rostal et al. (1998) e Robeck et al. (1990) em repteis. As características ultra-sonográficas dos testículos foram pouco acessadas, provavelmente sofrendo influência da maturidade sexual desses animais, o que, em algum momento, dificultou a caracterização e determinação precisa de suas dimensões. De igual modo, estes aspectos também foram abordados por Isaza et al. (1993a,b) em seus estudos para a mesma espécie.

\section{CONCLUSÕES}

O exame ultra-sonográfico demonstrou-se como uma técnica capaz de identificar as estruturas que compõem a cavidade celomática da jibóia (Boa constrictor). Esta técnica possibilitou identificar a morfologia, sintopia e aparência ultra-sonográfica de órgãos como o fígado e rins. Para este estudo, a influência da maturidade sexual determinou uma pobre visualização das estruturas reprodutivas nos machos, enquanto que nas fêmeas a técnica ultra-sonográfica permitiu acessar as estruturas componentes do seu trato reprodutivo, em maior ou menor grau, na dependência do seu ciclo reprodutivo.

\section{REFERÊNCIAS}

Alves F.R., Costa F.B., Arouche M.M.S., Barros A.C.E., Miglino M.A., Vulcano L.C. \& Guerra P. C. 2007. Ultrasonographic evaluation of the urinary system, liver and uterus of Cebus apella monkey. Pesq. Vet. Bras. 27(9):377-382.

Bertona M. \& Chiaraviglio M. 2003. Reproductive biology, mating aggregations, and sexual dimorphism of the argentine boa constrictor (Boa constrictor occidentalis). J. Herpet. 37(3):510-516.

Bizerra A., Marques O.A.V. \& Sazima I. 2005. Reproduction and feeding of the colubrid snake Tomodon dorsatus from south-eastern Brazil. Amphibia-Reptilia 26(1):33-38.

Bradshaw C.J.A., McMahon C.R. \& Hays G. 2007. Behavioral inference of diving metabolic rate in free-ranging leatherback turtles. Physiol. Bio-Chem. Zool. 80(2):209-219.

Bragdon D.E. 1953. A Contribution to the surgical anatomy of the water snake, Natrix sipedon-sipedon: The location of the visceral endocrine organs with reference to ventral scutellation. Anat. Rec. 117(2):145161.

Bragdon D.E. 1953. Lipid distribution in corpora-lutea of pregnancy in the water snake, Natrix sipedon-sipedon. Anat. Rec. 115(2):288-288.

Chetboul V., Schilliger L., Tessier D., Pouchelon J.L. \& Frye F. 2004. Specific features of echocardiographic examination in ophidians. Schweizer Archiv für Tierheilkunde 146(7):327-334.

Chiaraviglio M., Bertona M., Sironi M. \& Lucino S. 2003. Intrapopulation variation in life history traits of Boa constrictor occidentalis in Argentina. Amphibia-Reptilia 24(1):65-74.

Das B. \& Banerjee S. 2004. Inertial snake for contour detection in ultrasonography images. IEE Proceedings-Vision Image and Signal Processing 151(3):235-240.

De Sousa-e-Silva M.C.C., Tomy S.C., Tavares F.L., Navajas L., Larsson M.H.M.A., Lucas S.R.R., Kogika M.M. \& Sano-Martins I.S. 2003.
Hematological, hemostatic and clinical chemistry disturbances induced by Crotalus durissus terrificus snake venom in dogs. Human Exp. Toxicol. 22(9):491-500.

Fordham D.A., Georges A. \& Barry W.B. 2007. Demographic response of snake-necked turtles correlates with indigenous harvest and feral pig predation in tropical northern Australia. J. Anim. Ecol. 76(6):1231-1243.

Gartrell B.D., Girling J.E., Edwards A. \& Jones S.M. 2002. Comparison of noninvasive methods for the evaluation of female reproductive condition in a large viviparous lizard, Tiliqua nigrolutea. Zoo Biol. 21(3):253-268.

Gilman C.A. \& Wolf B.O. 2007. Use of portable ultrasonography as a nondestructive method for estimating reproductive effort in lizards. J. Exp. Biol. 210(11):1859-1867.

Gitirana L.D. 1987. The 1st report of the occurrence of Cilium in fatstoring cells in the reptilian liver (Eumeces-Algeriensis, Daudin 1802). Archivum Histologicum Japonicum 50(3):359-361.

Ibarguengoytia N.R. \& Cussac V.E. 1996. Reproductive biology of the viviparous lizard, Liolaemus pictus (Tropiduridae): Biennial female reproductive cycle? Herpet. J. 6(4):137-143.

Isaza R., Ackerman N. \& Jacobson E.L. 1993a. Ultrasound imaging of the celomic structures in the Boa constrictor (Boa-Constrictor). Vet. Radiol. Ultrasound. 34(6):445-450.

Isaza R., Ackerman N. \& Schumacher J. 1993b. Ultrasound-guided percutaneous liver-biopsy in snakes. Vet. Radiol. Ultrasound. 34(6): 452-454.

Loizou C.P., Pattichis C.S., Pantziaris M., Tyllis T. \& Nicolaides A. 2007. Snakes based segmentation of the common carotid artery intima media. Med. Biol. Eng. Comput. 45(1):35-49.

Madsen T., Ujvari B., Shine R. \& Olsson M. 2006. Rain, rats and pythons: Climate-driven population dynamics of predators and prey in tropical Australia. Aust. Ecology. 31(1):30-37.

Martinez-Torres M., Guzman-Rodriguez R., Cárdenas-Leon M. \& Brunner-Reynaldo N. 2006. Follicular development and ovulation determined by ultrasound imaging in the viviparous lizard Barisia imbricata (Reptilia: Anguidae). Southwestern Naturalist 51(3):401-406.

Nogueira C., Sawaya R.J. \& Martins M. 2003. Ecology of the pitviper, Bothrops moojeni, in the Brazilian Cerrado. J. Herpet. 37(4):653-659.

O'Donnell R.P., Shine R. \& Mason R.T. 2004. Seasonal anorexia in the male red-sided garter snake, Thamnophis sirtalis parietalis. Behavioral Ecology and Sociobiology 56(5):413-419.

Penninck D.G., Stewart J.S., Paul-Murphy J. \& Pion Paul. 1991. Ultrasonography of the California desert tortoise (Xerobates agassizi): Anatomy and application. Vet. Radiol. 32(3):112-116.

Robeck T.R., Rostal D.C., Burchfiel P.M., Owens D.W. \& Kraemer D.C. 1990. Ultrasound imaging of reproductive-organs and eggs in Galapagos tortoises, Geochelone-Elephantopus spp. Zoo Biol. 9(5):349359.

Roig J.M., Carretero M.A. \& Liorente G.A. 2000. Reproductive cycle in a pyrenean oviparous population of the common lizard (Zootoca vivipara). Netherlands J. Zool. 50(1):15-27.

Rostal D.C., Robeck T.R., Grumbles J.S., Burchfield P.M. \& Owens D.W. 1998. Seasonal reproductive cycle of the Galapagos tortoise (Geochelone nigra) in captivity. Zoo Biol. 17(6):505-517.

Rostal D.C. \& Robeck T.R. 1989. Seasonal cycle of testosterone and reproduction in captive Galapagos tortoises, Geochelone-Elephantopus. Am. Zool. 29(4):A97-A97.

Sainsbury A.W. \& Gili C. 1991. Ultrasonographic anatomy and scanning technique of the celomic organs of the Bosc Monitor (Varanus exanthematicus). J. Zoo Wildl. Med. 22(4):421-433.

Santos X. \& Llorente G.A. 2004. Lipid dynamics in the viperine snake, Natrix maura, from the Ebro Delta (NE Spain). Oikos 105(1):132-140.

Snyder P.S., Shaw N.G. \& Heard D.J. 1999. Two-dimensional echocardiographic anatomy of the snake heart (Python molurus bivittatus). Vet. Radiol. Ultrasound. 40(1):66-72.

Todd B.D. \& Andrews K.M. 2008. Response of a reptile guild to forest harvesting. Conserv. Biol. 22(3):753-761. 